\title{
Prediction of monthly mean daily global solar radiation using Artificial Neural Network
}

\author{
V Sivamadhavi ${ }^{1, *}$ and $\mathrm{R}$ SAmuel Selvaraj ${ }^{2}$ \\ ${ }^{1}$ Department of Physics, Bharathi Women's College, Chennai, Tamil Nadu, India. \\ ${ }^{2}$ Department of Physics, Presidency College, Chennai, Tamil Nadu, India. \\ ${ }^{*}$ Corresponding author. e-mail: siva_madhavi03@yahoo.com
}

In this study, a multilayer feed forward (MLFF) neural network based on back propagation algorithm was developed, trained, and tested to predict monthly mean daily global radiation in Tamil Nadu, India. Various geographical, solar and meteorological parameters of three different locations with diverse climatic conditions were used as input parameters. Out of 565 available data, 530 were used for training and the rest were used for testing the artificial neural network (ANN). A 3-layer and a 4-layer MLFF networks were developed and the performance of the developed models was evaluated based on mean bias error, mean absolute percentage error, root mean squared error and Student's $t$-test. The 3-layer MLFF network developed in this study did not give uniform results for the three chosen locations. Hence, a 4-layer MLFF network was developed and the average value of the mean absolute percentage error was found to be $5.47 \%$. Values of global radiation obtained using the model were in excellent agreement with measured values. Results of this study show that the designed ANN model can be used to estimate monthly mean daily global radiation of any place in Tamil Nadu where measured global radiation data are not available.

\section{Introduction}

Energy crisis is one of the most important challenges faced by many countries. In this scenario, solar energy plays a vital role as a renewable energy because of its unpolluted nature and its reliability in tropical countries like India. Tamil Nadu is one of the states located in the southern peninsular region of India lying in the sunny belt between $8.5^{\circ}$ and $13.35^{\circ} \mathrm{N}$. Its geographical location is advantageous for utilizing the solar energy. For effective and efficient utilization of solar energy, it is necessary to have a precise knowledge about the various components of solar energy available at the locations of our interest. Global radiation is the most important component of solar radiation since it gives the total solar availability at a given place. Global radiation is measured only at a few locations due to the high cost involved in the purchase of various equipments and maintenance thereof. In view of the above said factors, mathematical models become inevitable for places where measurement of global radiation is not done.

Various empirical models have been developed by many researchers using meteorological parameters such as sunshine hours, temperature, etc., to estimate monthly mean daily global radiation. Veeran and Kumar (1993) developed an Angstrom type regression model correlating global radiation and sunshine hours to estimate the monthly mean daily global radiation at two locations in India using the data of five years. Ahmad and Ulfat (2004) employed the regression technique and proposed Angstrom type empirical equation of first

Keywords. Artificial neural network; back propagation; global radiation; multilayer perceptron network; Tamil Nadu. 
and second order for determining the monthly mean daily global radiation at Karachi, Pakistan. Sabbagh et al. (1977) developed a regression model correlating the monthly mean daily global radiation with sunshine hours, relative humidity, maximum temperature, latitude and altitude for various places in Egypt, Kuwait, Lebanon, Sudan and Saudi Arabia. Chandal et al. (2005) correlated the monthly mean daily global radiation with temperature, sunshine hours, latitude and altitude for six Indian locations using the data of 1015 years. Augustine and Nnabuchi (2009) developed an Angstrom type correlation equation to predict monthly mean daily global solar radiation incident on a horizontal surface in Warri, Nigeria. Prieto et al. (2009) proposed an empirical model to estimate monthly mean daily global radiation using air temperature in Asturias, Spain. Ali (2008) modified three existing radiation models to estimate monthly mean daily global radiation in various cities in central arid desert of Iran by including the parameters such as altitude, Sun-Earth distance and number of dusty days. These models were developed only for specific locations and so they have limited applications.

Artificial neural network offers a better way to predict various components of solar radiation using many meteorological and geographical parameters as input. The basic assumption underlying the ANN models is that there exists a non-linear relationship between solar radiation and these parameters. Use of ANNs has gained increasing popularity for applications where a mechanistic description of the dependency between dependent and independent variables is either unknown or very complex (Almeida 2002). ANN is one of the best tools for non-linear mapping. ANN technique was previously used to predict the components of solar radiation such as hourly diffuse radiation (Soares et al. 2004; Alam et al. 2009), hourly direct radiation (Lopez et al. 2005), hourly global radiation (Krishnaiah et al. 2007), and daily global radiation (Mohandes et al. 1998; Reddy and Ranjan 2003; Fadare et al. 2010).

Global radiation is the most important component of solar radiation. Fadare et al. (2010) studied the feasibility of an ANN based model for the prediction of monthly mean daily global radiation at various locations in Africa. Mohandes et al. (1998) used a multilayer perceptron neural network for modelling monthly mean daily global radiation in the Kingdom of Saudi Arabia. So far, there has been only one study on the modelling of global radiation in India using ANN technique (Reddy and Ranjan 2003) where the maximum mean percentage error is $10.2 \%$ and $12.5 \%$ for the stations, Mangalore and New Delhi, respectively. Reddy and Ranjan (2003) developed a Multi Layer Feed
Forward network for the estimation of monthly mean daily and hourly values of global radiation in India using various geographical and meteorological parameters. The ANN model employed in their study contains two hidden layers with eight and seven neurons respectively. The results of their study justify the application of artificial neural networks, the most sophisticated non-linear model for modelling global radiation.

The main objective of our study is to develop a computationally simpler ANN model to estimate global radiation with mean absolute percentage error of less than 5\% using commonly measured weather parameters for places in Tamil Nadu. Solar parameters such as solar declination, sunrise hour angle, extraterrestrial radiation and maximum possible sunshine duration (day length) have been used as inputs along with some geographical and meteorological parameters. The inputs used in our study are readily available in all meteorological stations. In the present study, a multilayer feed forward neural network based on the Lavenberg-Marquardt back propagation learning algorithm was developed, trained and tested using the geographical, solar and meteorological parameters of three locations in Tamil Nadu with varying climatic conditions.

\section{Materials and methods}

\subsection{ANN model}

Artificial neural networks mimic the biological neural networks. ANN models have been used for various applications including pattern recognition, pattern classification, non-linear mapping, prediction and simulation. Among the different types of networks available, we chose a multilayer perceptron (MLP) network. A 3-layer multilayer feed forward (MLFF) network as shown in figure 1 and a 4-layer MLFF network as shown in figure 2 based on back propagation algorithm were developed, trained and tested for estimating monthly mean daily global radiation using the geographical, solar and meteorological parameters.

Design and training parameters of our network are shown in table 1 . Fourteen neurons in the input

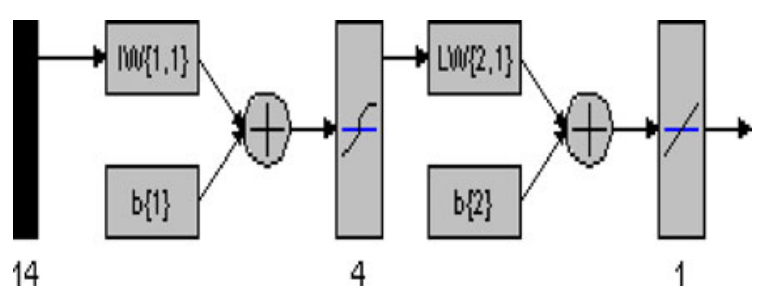

Figure 1. Topology of 3-layer MLFF Network. 
layer correspond to the following input parameters with units in parentheses:

1. Month number

2. Latitude (deg)

3. Longitude (deg)

4. Altitude (m)

5. Solar declination (radian)

6. Sunrise hour angle (radian)

7. Day length (hour)

8. Extraterrestrial radiation on a horizontal surface $\left(\mathrm{MJ} /\right.$ day $\left./ \mathrm{m}^{2}\right)$

9. Sunshine duration (hour)

10. Maximum temperature $\left({ }^{\circ} \mathrm{C}\right)$

11. Minimum temperature $\left({ }^{\circ} \mathrm{C}\right)$

12. Relative Humidity at 8:30 hrs IST (\%)

13. Relative Humidity at 17:30 hrs IST (\%) and

14. Wind speed (km/hour).

Monthly mean daily values of these parameters were given as inputs to our network. Single neuron in the output layer corresponds to the output parameter, monthly mean daily global radiation on a horizontal surface.

Back propagation network is a supervised learning network, i.e., a network that learns with a teacher. The network is trained with a training data that consists of an input vector set and a

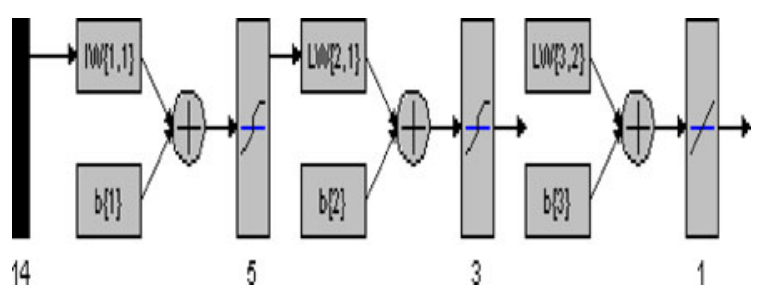

Figure 2. Topology of 4-layer MLFF Network. target vector set. While training the network, weights and biases are so adjusted that the error between the target and the predicted output vectors is minimized. In the first phase of the training, the input vectors are propagated in the forward direction from the input layer to the output layer (called the Forward phase) and in the second phase, the error is propagated in the backward direction (called the Backward phase) to update the weights minimizing the errors.

Lavenberg-Marquardt back propagation algorithm (LM algorithm) was used for training the ANN model. LM algorithm is a variation of Newton's method. This algorithm provides a nice compromise between the speed of Newton's method and guaranteed convergence of steepest descent algorithm. The key step in LM algorithm is the computation of Jacobian matrix. In the standard back propagation algorithm, first derivatives of squared errors with respect to weights and biases are computed. In LM algorithm, Jacobian matrix is constructed by computing first derivatives of the errors themselves. The step by step procedure of LM algorithm can be summarized as follows (Hagan and Menhaj 1994):

Step 1. The input vector is presented to the network. The outputs of the layers are computed using the equations (1) and (2).

$$
\begin{gathered}
\mathbf{y}^{1}=\mathbf{p} \\
\mathbf{y}^{n}=\mathrm{f}^{n}\left[\mathbf{W}^{n} \mathbf{y}^{n-1}+\mathbf{b}^{n}\right](n=2,3, \ldots, M)
\end{gathered}
$$

Here, $\mathbf{p}$ represents the input vector; $\mathbf{y}^{1}$ and $\mathbf{y}^{n}$ represent the output vector of first and $n$th layers respectively; $\mathbf{W}^{n}$ and $\mathbf{b}^{n}$ are the weight matrix and the bias vector of the $n$th layer respectively; $M$ represents the total number of layers in the

\begin{tabular}{|c|c|c|c|}
\hline Sl. no. & Parameter & Selected value & Remarks \\
\hline 1 & No. of hidden layers & 1 and 2 & $\begin{array}{l}\text { Simplest and next simpler } \\
\text { models were selected }\end{array}$ \\
\hline 2 & No. of hidden neurons & 4,5 and 3 & $\begin{array}{l}\text { Chosen such that the it minimizes the } \\
\text { error and avoids over fitting }\end{array}$ \\
\hline 3 & Activation function - Hidden layers & 'tansig' & $\mathrm{F}(\mathrm{x})=2 /(1+\exp (-2 \mathrm{x}))-1$ \\
\hline 4 & Activation function - the output layer & 'purelin' & $\mathrm{F}(\mathrm{x})=\mathrm{x}$ \\
\hline 5 & Training function & trainlm & $\begin{array}{l}\text { Levenberg-Marquardt back } \\
\text { propagation algorithm }\end{array}$ \\
\hline 6 & Learning function & learngdm & $\begin{array}{l}\text { Gradient descent with momentum learning function } \\
\text { to update the weights/biases. }\end{array}$ \\
\hline 7 & Learning rate & 0.001 & Adaptive learning rate algorithm was used \\
\hline 8 & Performance function & msereg & Chosen to avoid over fitting \\
\hline 9 & Goal & 0.0001 & \\
\hline 10 & Epochs & 1000 & \\
\hline
\end{tabular}

Table 1. Design and training parameters of the ANN model. 
network; and $\mathrm{f}^{n}$ represents the activation function of the $n$th layer.

Step 2. The sum of the squared error and the error vector are computed using equations (3) and (4).

$$
\begin{aligned}
& V=\sum_{j=1}^{N}\left[\sum_{i=1}^{K}\left(d_{i j}-a_{i j}\right)^{2}\right]
\end{aligned}
$$

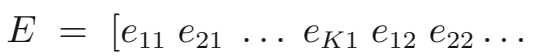

$$
\begin{aligned}
& \left.\begin{array}{llllll}
e_{K 2} & \ldots & e_{1 N} & e_{2 N} & \ldots & e_{K N}
\end{array}\right]^{T} .
\end{aligned}
$$

Here, $K$ is the number of neurons in the output layer; $N$ is the number of samples in the input-output pair; $d_{i j}$ and $a_{i j}$ represent the desired output and the actual output of the $i$ th neuron corresponding to the $j$ th sample of the training data; and $e_{i j}$ represents the error corresponding to the $i$ th neuron and $j$ th sample and is given by:

$$
e_{i j}=d_{i j}-a_{i j}
$$

Step 3. The Jacobian matrix is constructed using the equation

$$
\mathbf{J}=\left[\begin{array}{cccc}
\frac{\partial e_{11}}{\partial x_{1}} & \frac{\partial e_{11}}{\partial x_{2}} & \cdots & \frac{\partial e_{11}}{\partial x_{P}} \\
\frac{\partial e_{21}}{\partial x_{1}} & \frac{\partial e_{21}}{\partial x_{2}} & \cdots & \frac{\partial e_{21}}{\partial x_{P}} \\
\vdots & \vdots & \vdots & \\
\frac{\partial e_{k 1}}{\partial x_{1}} & \frac{\partial e_{k 1}}{\partial x_{2}} & \cdots & \frac{\partial e_{k 1}}{\partial x_{P}}
\end{array}\right]
$$

where $\mathbf{X}=\left[\begin{array}{llll}\mathrm{x}_{1} & \mathrm{x}_{2} & \ldots & \mathrm{x}_{\mathrm{P}}\end{array}\right]^{\mathrm{T}}$ is the vector of adjustable parameters, i.e., weights and biases.

Step 4. The weights and biases are adjusted according to the equation

$$
\Delta \mathbf{X}=\left[\mathbf{J}^{\mathbf{T}} \mathbf{J}+\mu \mathbf{I}\right]^{-1} \mathbf{j}^{\mathbf{T}} \mathrm{E}
$$

where $\mathbf{I}$ is the identity matrix and $\mu$ is the learning parameter.

Step 5. The sum of squared errors is recomputed using the updated weights and biases. If the new value is smaller than the previous value of the sum of squared errors, then $\mu$ is divided by a factor of $\beta$ and the steps 15 are repeated. If the new value of sum of squared errors is higher than its previous value, then $\mu$ is multiplied by a factor of $\beta$ and steps $4-5$ are repeated. We have used $\mu=0.001$ and $\beta=10$.
One of the problems that arises while training a neural network is 'over fitting' which results in large error on testing data and very small error on training data. In this case, the network has memorized the training set values and it has not learned to generalize. The network must be trained to generalize. This can be achieved in many ways. One way is to design the network such that the number of adjustable parameters (weights and biases) is much smaller than the total number of data points used for training (Hagan et al. 1996). In the MLFF network, the number of neurons in the input layer, output layer and the hidden layers should satisfy the inequality (equations 8 and 9) (Salai Selvam and Shelbagadevi 2010).

$$
\begin{aligned}
& N_{\mathrm{i}} N_{\mathrm{h} 1}+N_{\mathrm{h} 1}+N_{\mathrm{h} 1} N_{\mathrm{o}}+N_{\mathrm{o}} \leq N \\
& \quad(\text { for 3-layer MLFF network) }
\end{aligned}
$$

$$
N_{\mathrm{i}} N_{\mathrm{h} 1}+N_{\mathrm{h} 1}+N_{\mathrm{h} 1} N_{\mathrm{h} 2}+N_{\mathrm{h} 2}+N_{\mathrm{h} 2} N_{\mathrm{o}}+N_{\mathrm{o}} \leq N
$$

(for 4-layer MLFF network)

where $N_{\mathrm{i}}, N_{\mathrm{h} 1}, N_{\mathrm{h} 2}$ and $N_{\mathrm{o}}$ are the number of neurons in input layer, hidden layer-1, hidden layer-2 and output layer, respectively and $N$ is the number of data points used for training.

In the designed 3-layer MLFF network with four hidden neurons, the number of adjustable parameters came out to be 65 . In our 4-layer MLFF network, we chose $N_{\mathrm{h} 1}$ and $N_{\mathrm{h} 2}$ to be 5 and 3 , respectively resulting in the number of adjustable parameters to be 97 . The size of the training data is 530. Hence the designed network can generalize itself. Another way to improve the generalization is to adopt 'regularization' which involves modifying the performance function of the network from 'mse' to 'msereg'. This was also done to ensure that the network generalizes well. Here, msereg = $\alpha$ mse $+(1-\alpha)$ msw, where mse and msw are mean squared values of errors and weights; $\alpha$ is a parameter which determines the relative significance of mse and msw.

The data were normalized using equation (10) to fall in the interval $[-1,+1]$ to give numerical stability to the network.

$$
\left.D_{n}=\left[2\left(D-D_{\min }\right) / D_{\max }-D_{\min }\right)\right]-1
$$

where $D$ and $D_{n}$ are the actual and the normalized data respectively; $D_{\max }$ and $D_{\min }$ are the maximum and minimum values of the actual data. The implementation of the ANN model was done using MATLAB. Training is terminated when the set goal value of performance function is reached or the total number of epochs is completed. MATLAB code for the implementation of ANN model used 
Table 2. Stations used for the study.

\begin{tabular}{|c|c|c|c|c|c|c|}
\hline Sl. no. & $\begin{array}{l}\text { Climatic } \\
\text { zone }\end{array}$ & Station & $\begin{array}{l}\text { Lattitude } \\
\qquad\left(^{\circ}\right)\end{array}$ & $\begin{array}{c}\text { Longitude } \\
\left({ }^{\circ}\right)\end{array}$ & $\begin{array}{l}\text { Altitude } \\
\quad(\mathrm{m})\end{array}$ & $\begin{array}{c}\text { No. of data } \\
\text { points (month) }\end{array}$ \\
\hline 1 & Hill station & Kodaikanal & 10.2333 & 77.4667 & 2345 & 190 \\
\hline 2 & Coastal area & Chennai & 13 & 80.1833 & 16 & 342 \\
\hline 3 & Continental area & Coimbatore & 11 & 77 & 409 & 33 \\
\hline
\end{tabular}

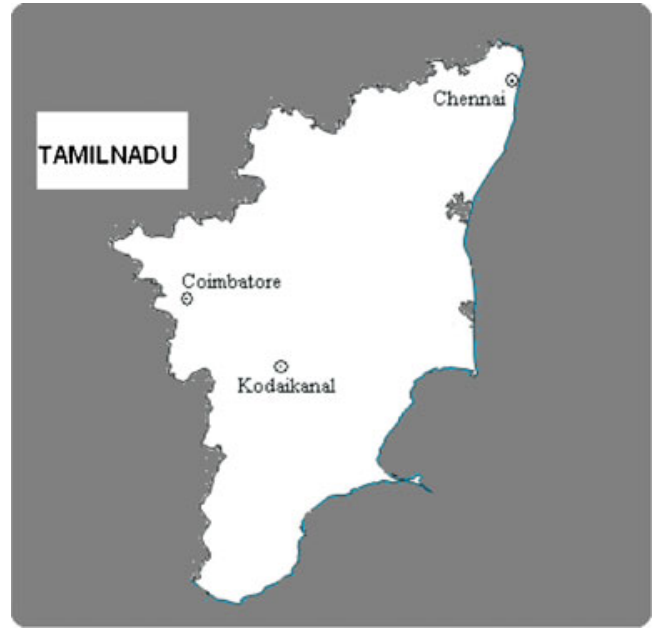

Figure 3. Location map of study sites.

in the present study shall be made available to the interested readers by the authors on request.

\subsection{Data}

The monthly mean daily values of global radiation on a horizontal surface, sunshine hours, relative humidity at 8:30 IST, relative humidity at 17:30 IST, minimum temperature, maximum temperature and wind speed are the meteorological parameters used in this study. They have been obtained from National Data Center, India Meteorological Department, Pune, India and Regional Meteorological Center, Chennai, India for three locations each from a different climatic zone. The geographical parameters of these stations are given in table 2 . The location map of the study sites is shown in figure 3.

The training set is normally a representative of a much larger class of population. The network can generalize well if the training set consists of members of each class in the total population. This forms the basis to obtain the data from three different climatic regions. Any new location from Tamil Nadu can be categorized under any of the three categories used in our study. Hence, the model developed in the present study can be used to
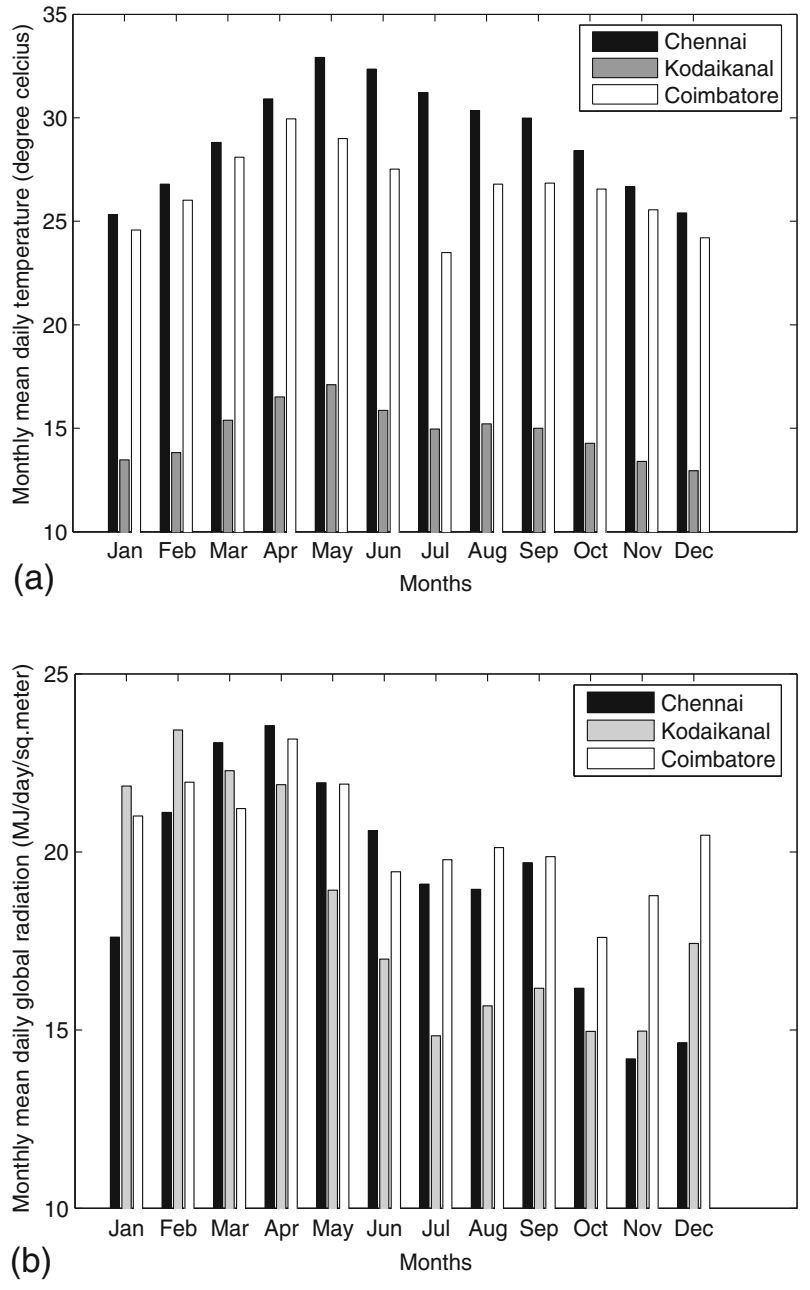

Figure 4. (a) Monthly mean daily temperature of the study sites and (b) monthly mean daily global radiation of the study sites.

estimate monthly mean daily global radiation at any location in Tamil Nadu where the measurement of global radiation is not done. Figure 4(a and b) shows how the three study sites vary in terms of monthly mean daily values of temperature and global radiation respectively.

The above-mentioned meteorological parameters have been obtained for a period of 342 months (1980 to 2009) for Chennai, of which the data of 2009 (12 months) have been used for testing and the rest (330) were used for training the network. For Kodaikanal, the global radiation data 
were available for 190 months during the period from 1984 to 2009 with many data gaps though the other meteorological parameters were available for the entire period. Of these, the data of 2009 (12 months) have been used for testing and the rest (178) were used for training the network. At Coimbatore, the measurement of global radiation was started by India Meteorological Department in the year 2006. Hence, the data for 33 months during the period from 2006 to 2008 were only available, of which the data of 2008 (11 months) were used for testing and the rest (22) were used for training.

Solar parameters used as input to the ANN model are the monthly mean daily values of solar parameters such as solar declination, sunrise hour angle, day length and the extraterrestrial radiation on a horizontal surface. They can be obtained from the following equations (Cooper 1969):

$$
\begin{gathered}
\delta=23.45 \sin [360(284+n) / 365] \\
\omega=\cos ^{-1}[-\tan \varphi \tan \delta] \\
S_{0}=(2 / 15) \omega \\
H_{d}=(24 / \pi) I_{0}[1.0+0.033 \cos (360 n / 365)] \\
\times[(\pi \omega / 180) \sin \varphi \sin \delta \\
+\cos \varphi \cos \delta \sin \omega]
\end{gathered}
$$

where $H_{d}$ is daily extraterrestrial radiation on a horizontal surface on the $n$th day, $I_{0}$ is hourly extraterrestrial radiation, $n$ is the day of the year starting from first January, $\omega$ is the sunrise hour angle on $n$th day, $\varphi$ is the latitude of the location of our interest, $\delta$ is the solar declination on $n$th day.

\subsection{Performance evaluation methods}

The performance of the proposed model has been evaluated based on the following well established statistical error parameters (Mac and Iqbal 1984):

(i) Mean Bias Error (MBE) defined as:

$$
\operatorname{MBE}=(1 / K) \sum\left(H_{\mathrm{cal}}^{i}-H_{\mathrm{obs}}^{i}\right)
$$

where $K$ is the total number of observations, $H_{\mathrm{obs}}^{i}$ and $H_{\mathrm{cal}}^{i}$ are the $i$ th observed value and $i$ th calculated value of global radiation.

(ii) Root Mean Square Error (RMSE) defined as:

$$
\operatorname{RMSE}=\left[(1 / K) \sum\left(H_{\mathrm{cal}}^{i}-H_{\mathrm{obs}}^{i}\right)^{2}\right]^{1 / 2}
$$

(iii) Mean Absolute Percentage Error (MAPE) defined as:

$$
\operatorname{MAPE}=(100 / K) \sum\left(\left(H_{\mathrm{cal}}^{i}-H_{\mathrm{obs}}^{i}\right) / H_{\mathrm{obs}}^{i}\right)
$$

The test of MBE provides information on the long term performance of the models. A positive MBE gives the average amount of overestimation in the predicted values and a negative MBE value gives the average amount of underestimation in the predicted values. One drawback of this test is that overestimation in one observation will cancel the underestimation in another observation. The MAPE avoids this error cancellation problem. But, normally this test parameter is not preferred in the analytical point of view because the absolute value of error is not a differentiable quantity. The test on RMSE provides information on the short term performance of the models. It allows a term by term comparison of the actual deviation between the calculated and the measured values. It is always positive (Jamil Ahmad and Tiwari 2010). Apart from these statistical tests, the $t$-statistic test proposed by Stone (1993) was also employed to assess the proposed models. This method has the added advantage of telling whether the model's estimates are statistically significant or not at a particular confidence level. The $t$-statistic $(T S)$ defined by Stone (1993) is as follows:

$T S=\left[(K-1)(\mathrm{MBE})^{2} /\left(\mathrm{RMSE}^{2}-\mathrm{MBE}^{2}\right)\right]^{1 / 2}$

A two tailed $t$-test was carried out at the $95 \%$ confidence level. TS should lie within the interval defined by $-T_{c}$ and $+T_{c}$ where $T_{c}$, the critical $T S$ value is obtained from the Student's distribution at desired confidence level with $(K-1)$ degrees of freedom (Daniel and Terrell 1992). The smaller the value of $T S$ the better is the performance of the model.

\section{Results and discussion}

Initially a 3-layer MLFF network with 4 hidden neurons was developed, trained and tested. The MBE, RMSE, MAPE and TS values obtained considering the three study sites individually and together are shown in figure $5(\mathrm{a}-\mathrm{d})$ for both training and testing phases. The MAPE obtained for the three climatic regions were not convincingly uniform and also that obtained for all the three climatic regions together was larger than our goal of obtaining MAPE less than 5\%. Hence, we moved on to 4-layer MLFF network (two hidden layers). 

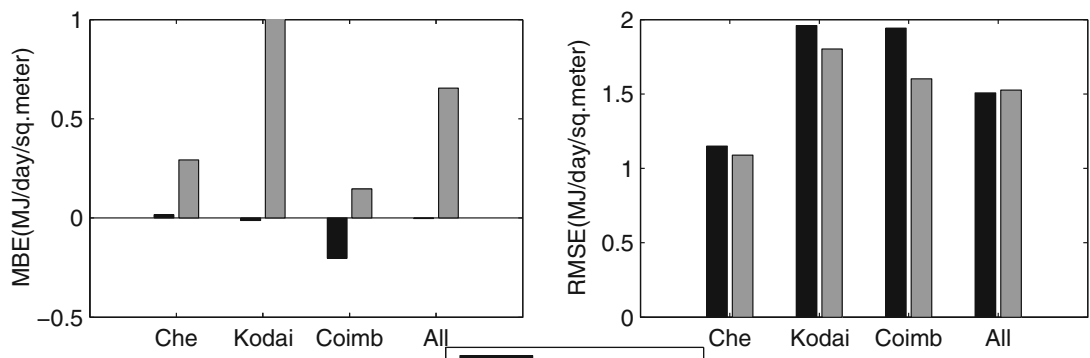

(a)

Training period Testing period

(b)

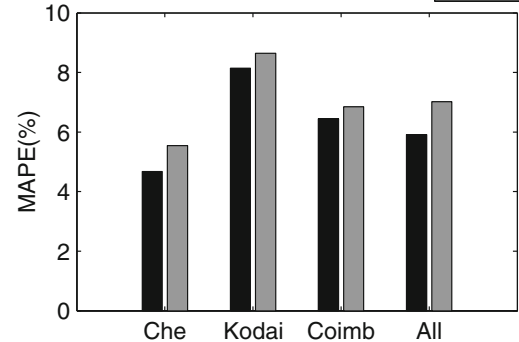

(c)

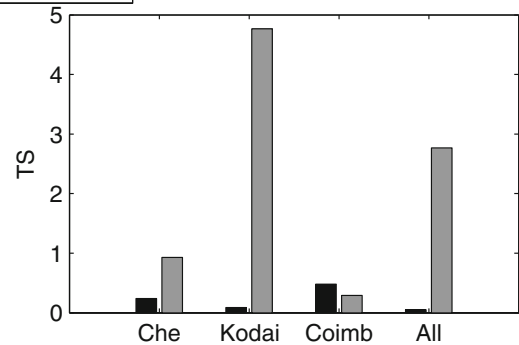

(d)

Figure 5. Performance evaluation of 3-layer MLFF network (a) MBE values, (b) RMSE values, (c) MAPE values, and (d) TS values.

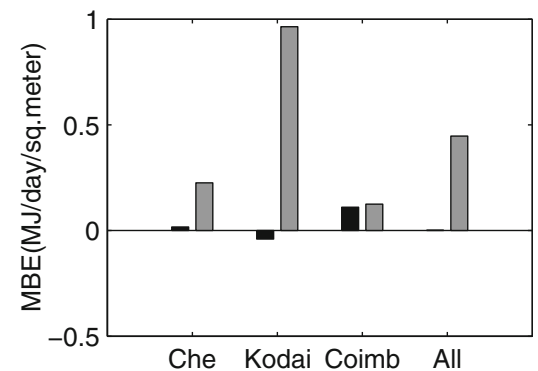

(a)

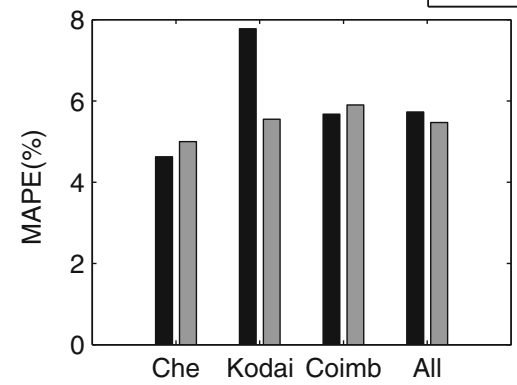

(c)

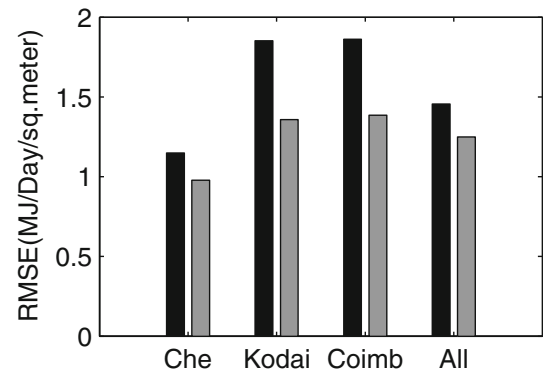

(b)

Training period Testing period

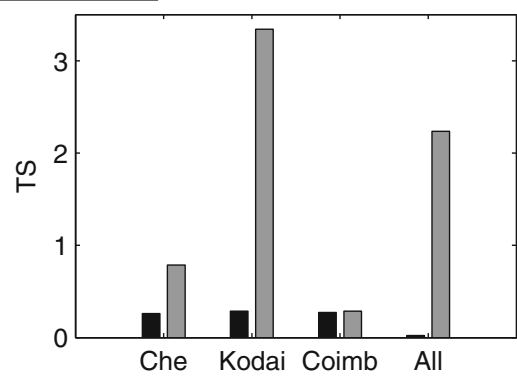

(d)

Figure 6. Performance evaluation of 4-layer MLFF network (a) MBE values, (b) RMSE values, (c) MAPE values, and (d) TS values.

Further discussions will be focused on the results of the 4-layer MLFF network only. The values of performance evaluation factors such as MBE, RMSE, MAPE and TS obtained with the 4-layer MLFF network are given in figure $6(\mathrm{a}-\mathrm{d})$. The
MAPE values obtained for the three locations are uniform. Values of certain error parameters for the testing phase are slightly higher than that of the training phase. This indicates that the ANN model proposed in this study has generalized well. 
In Student's $t$-test, the mean of predicted series is compared with the mean of measured series. In a two-tailed $t$-test, the possibility of deviation in either direction is tested. At the significance level of 0.05 , half of this value, i.e., 0.025 is used to test the significance in one direction and the other half is used to test the significance in the other direction. Hence, the critical value of the test statistic at 0.025 significance level has been obtained from the standard statistical table at $(K-1)$ degrees

Table 3. Comparison of TS values with $T_{c}$.

\begin{tabular}{lcc}
\hline Location & $T_{c}$ & $\begin{array}{c}\text { TS (4-layer } \\
\text { MLFF network) }\end{array}$ \\
\hline Chennai & 2.2010 & 0.7867 \\
Kodaikanal & 2.2010 & 3.3414 \\
Coimbatore & 2.2282 & 0.2871 \\
Overall & 2.0322 & 2.2362 \\
\hline
\end{tabular}
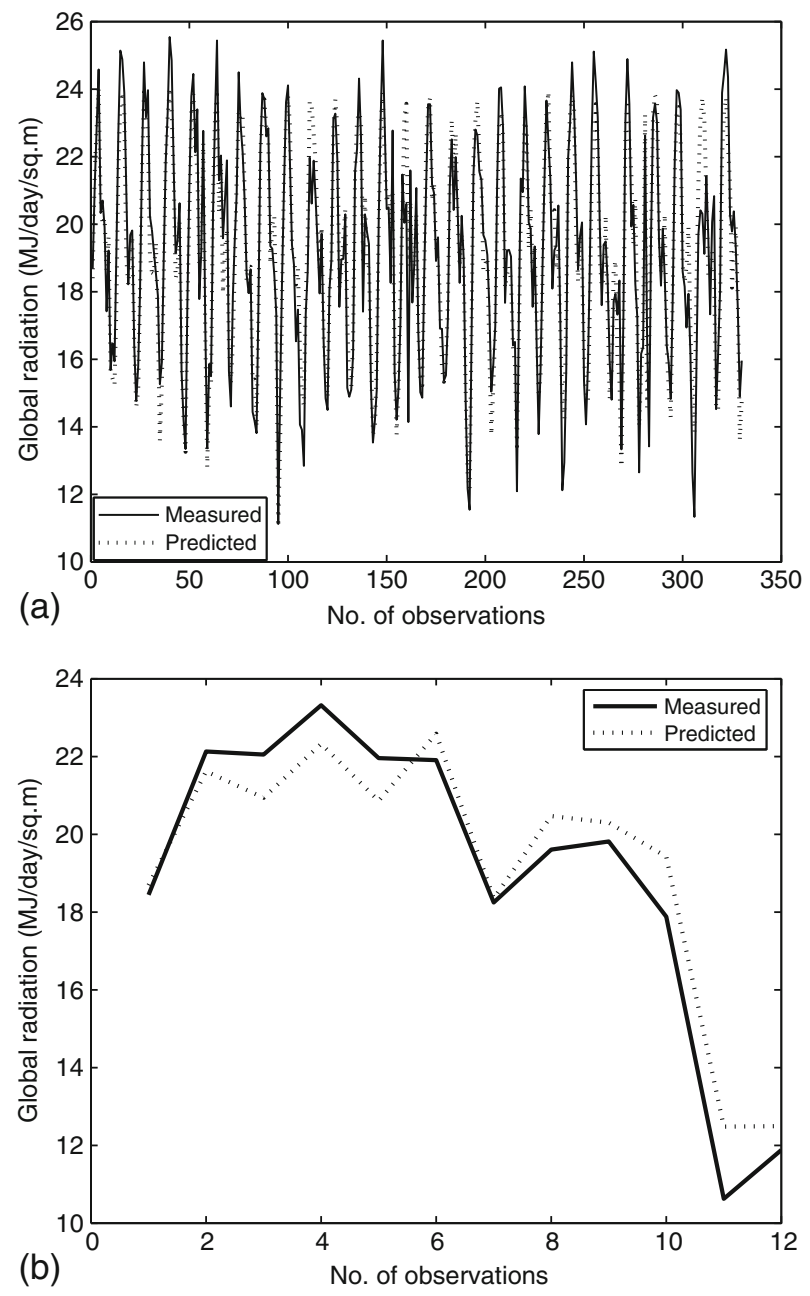

Figure 7. Measured and predicted values of monthly mean daily global radiation at Chennai (a) training phase and (b) testing phase. of freedom. $K=12$ in the case of Chennai and Kodaikanal, $K=11$ for Coimbatore, and $K=35$ for the overall model. Estimated $T S$ values for the testing phase are compared with the respective critical values in table 3 . The $T S$ values for Chennai and Coimbatore lie within the critical limit. However, the $T S$ value of Kodaikanal lies outside the critical limit. Kodaikanal is a high altitude station. The greenhouse warming in such places is much lower than that in other places. The role of greenhouse gases in the surface radiation budget is not included in our study. We have not used the concentration of greenhouse gases as input in the ANN model. This could be the possible cause for the higher value of $T S$ for Kodaikanal. Due to this, the $T S$ value obtained for all the three stations together also lies outside the critical limit.

Figure 7(a and b) shows how well the predicted values of monthly mean daily global radiation at Chennai agree with the measured values during
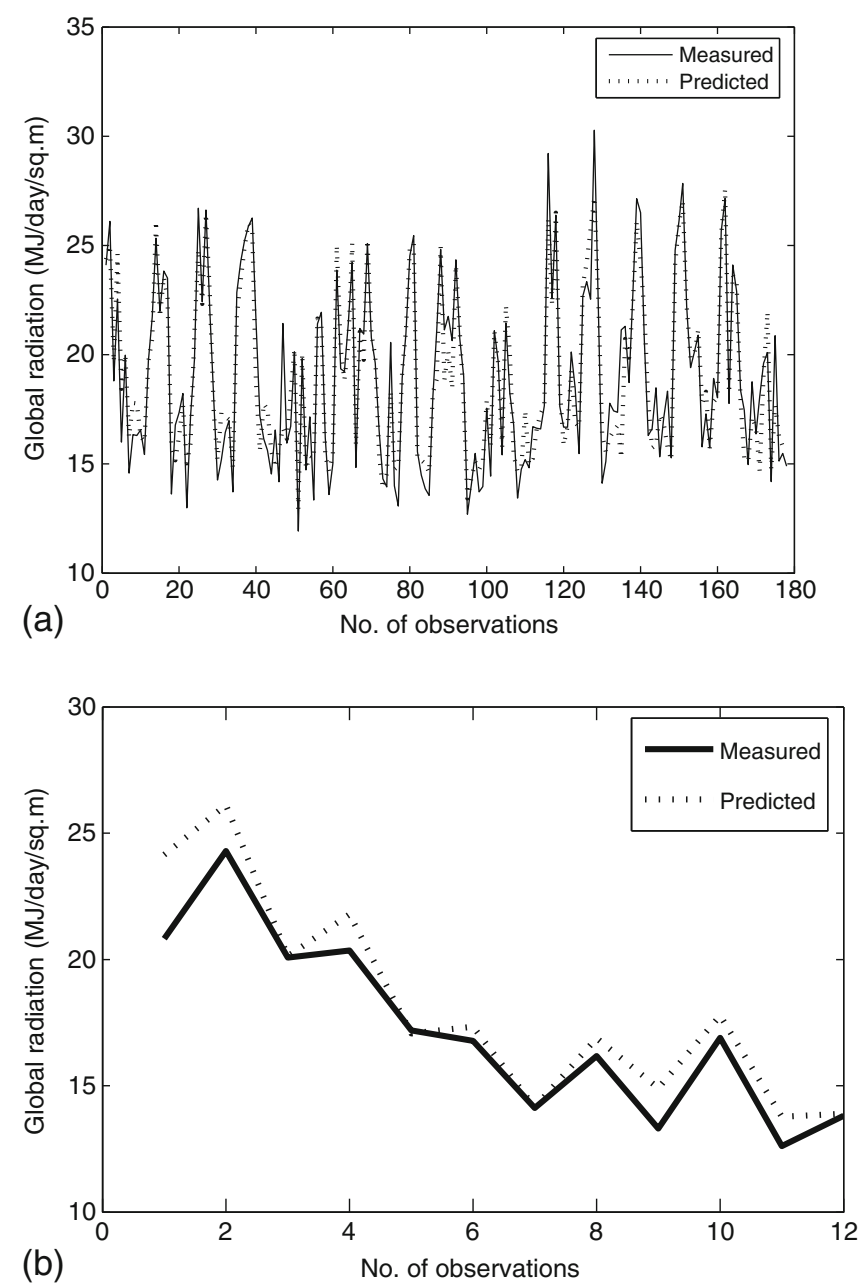

Figure 8. Measured and predicted values of monthly mean daily global radiation at Kodaikanal (a) training phase and (b) testing phase. 

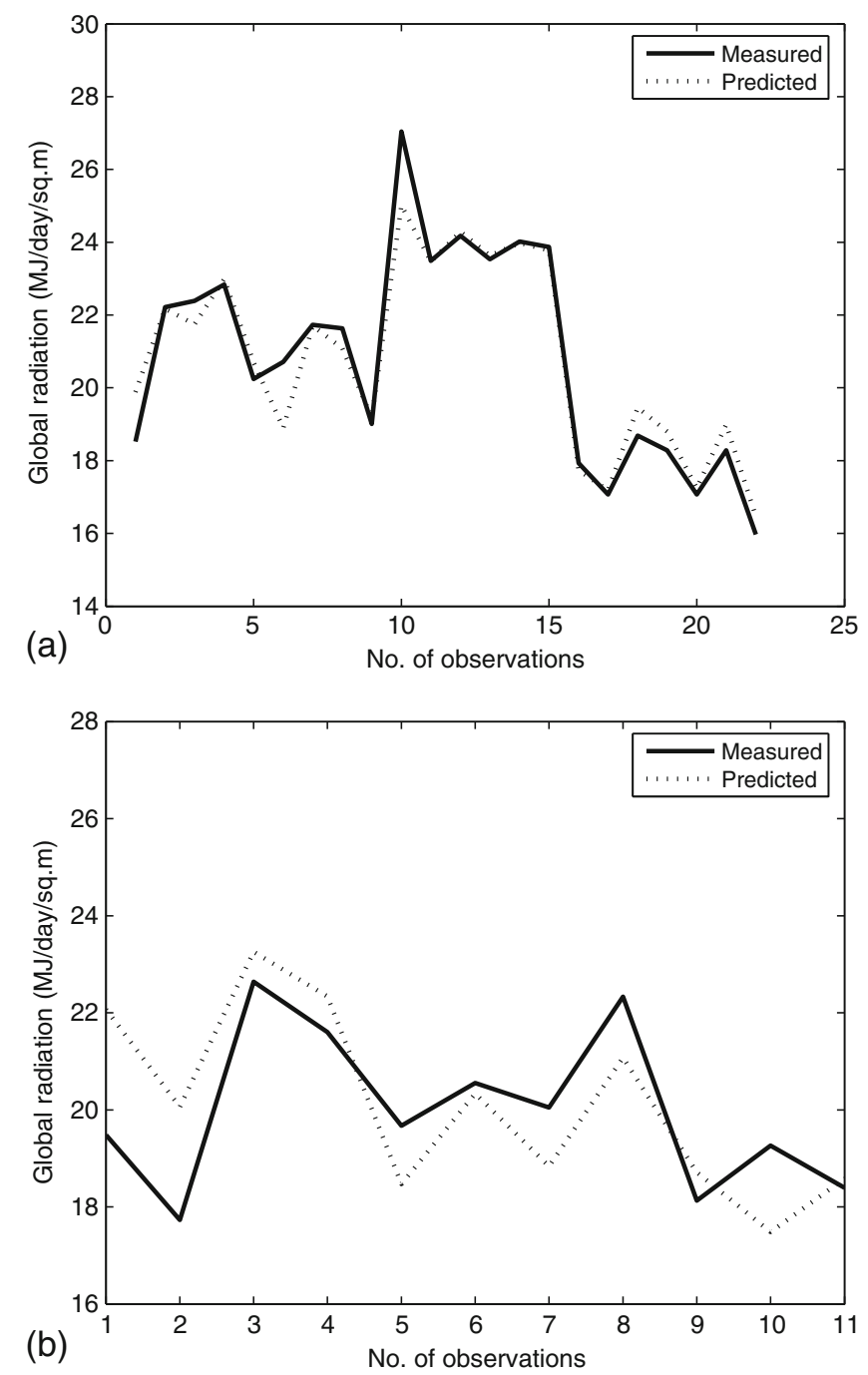

Figure 9. Measured and predicted values of monthly mean daily global radiation at Coimbatore (a) training phase and (b) testing phase.

training and testing phases. Comparison of predicted and measured values of global radiation for Kodaikanal and Coimbatore are shown in figures $8(\mathrm{a}$ and $\mathrm{b})$ and $9(\mathrm{a}$ and $\mathrm{b})$, respectively.

\section{Conclusion}

The uniform values of MAPE indicate that the model proposed in this study has generalized the training data of all the three locations equally well. The locations taken for this study have different climatic conditions. The ANN model has been trained to generalize for any new locations with similar climatic conditions as these three stations. Hence, this model can be used to predict the monthly mean daily global radiation at any location in Tamil Nadu.

\section{Acknowledgements}

Authors would like to convey their sincere gratitude to Dr K S Reddy, Indian Institute of Technology, Chennai, India for having given some valuable suggestions regarding the presented work. They also wish to express their sincere gratitude to the reviewers for the valuable suggestions which enabled to improve the manuscript. They thank the colleagues in the Department of English who helped in improving the presentation.

\section{References}

Ahmad F and Ulfat I 2004 Empirical model for the correlation of monthly average daily global solar radiation with hours of sunshine on a horizontal surface at Karachi, Pakistan; Turk. J. Phys. 28 301-307.

Alam S, Kaushik S C and Garg S N 2009 Assessment of diffuse solar energy under general sky condition using artificial neural network; Appl. Ener. 86 554-564.

Ali A S 2008 A simple formula for estimating global solar radiation in central arid deserts of Iran; Ren. Energy 33 1002-1010.

Almeida J S 2002 Predictive non-linear modeling of complex data by artificial neural networks; Curr. Opin. Biotechnol. 13 72-76.

Augustine C and Nnabuchi M N 2009 Correlation between sunshine hours and global solar radiation in Warri, Nigeria; The Pacific J. Sci. Tech. 10 574-579.

Chandal S S, Agarwal R K and Pandey A N 2005 New correlation to estimate global radiation on horizontal surface using sunshine hours and temperature data for Indian cities; J. Solar Energy Eng. 127 417-420.

Cooper P I 1969 The absorption of solar radiation in solar stills; Solar Energy 12 333-346.

Daniel W W and Terrell J C 1992 Business statistics for management and economics; 6th edn, London, Houghton Mifflin Company, p. A53.

Fadare D A, Irimisose I, Oni A O and Falana A 2010 Modeling of solar energy potential in Africa using an artificial neural network; Am. J. Sci. Indus. Res. 1 144-157.

Hagan M T and Menhaj M B 1994 Training feedforward networks with the Marquardt algorithm; IEEE Trans. Neural Networks 5 989-993.

Hagan M T, Demuth H B and Beale M 1996 Neural Network Design, PWS Publishing Company, Boston Ch. 2, 10, 11 $\& 12$.

Jamil Ahmad M and Tiwari G N 2010 Solar radiation models-review; Int. J. Energy Environ. 1 513-532.

Krishnaiah T, Srinivasa Rao S, Madhumurthy K and Reddy K S 2007 Neural network approach for modeling global solar radiation; J. Appl. Sci. Res. 3 1105-1111.

Lopez G, Batles F J and Tovar-Pescador J 2005 Selection of input parameters to model direct solar irradiance by using artificial neural networks; Energy 30 1675-1684.

Mac C and Iqbal M 1984 Statistical comparison of solar radiation correlation of monthly average global and diffuse radiation on horizontal surfaces; Solar Energy $\mathbf{3 3}$ 143-148.

Mohandes M, Rehman S and Halawani T 1998 Estimation of global solar radiation using artificial neural networks; In: Sixth Arab International Solar Energy Conference, Muscat, Sultanate of Oman, 29th March-1st April. 
Prieto J I, Martinez Garcia J C and Garcia D 2009 Correlation between global solar irradiation and air temperature in Asturias, Spain; Solar Energy 83 1076-1085.

Reddy K S and Ranjan M 2003 Solar resource estimation using artificial neural networks and comparison with other correlation models; Ener. Conver. Manag. 44 2519-2530.

Sabbagh J A, Sayigh A A M and El-Salam E M A 1977 Estimation of the total solar radiation from meteorological data; Solar Energy 19 307-311.

Salai Selvam V and Shelbagadevi S 2010 Classification of characteristic waves of sleep EEG using first and higher order statistics and Neural Network; CiiT International
Journal of Artificial Intelligent Systems and Machine Learning 2 142-150.

Soares J, Oliveira A P, Boznar M Z, Mlakar P, Escobedo J F and Machado A J 2004 Modeling hourly diffuse solar radiation in the city of Sao Paulo using a neural network technique; Appl. Energy 79 201-214.

Stone R J 1993 Improved statistical procedure for evaluation of solar radiation estimation models; Solar Energy $51289-291$.

Veeran P K and Kumar S 1993 Analysis of monthly average daily global radiation and monthly average sunshine duration at two tropical locations; Ren. Energy $\mathbf{3}$ 935-939.

MS received 17 August 2011; 27 April 2012; accepted 30 April 2012 